\title{
A genetically trained adaptive neuro-fuzzy inference system network utilized as a proportional-integral-derivative-like feedback controller for non-linear systems.
}

\begin{abstract}
This paper presents a genetically trained PID (proportional-integral-derivative)-like ANFIS (adaptive neuro-fuzzy inference system) acting as a feedback controller to control non-linear systems. Three important issues are addressed in this paper, which are, first, the evaluation of the ANFIS as a PID-like controller; second, the utilization of the GA (genetic algorithm) alone to train the ANFIS controller, instead of the hybrid learning methods that are widely used in the literature; and, third, the determination of the input and output scaling factors for this controller by the GA. The GA, with real-coding operators, is used to adjust all of the ANFIS parameters, which include the input and output scaling factors, the centres and widths of the input membership functions (MFs), and the consequent parameters. To show the effectiveness of this controller and its learning method, several non-linear plants, including the CSTR (continuous stirred tank reactor), have been selected to be controlled by this controller through simulation. Moreover, this controller's robustness to output disturbances has also been tested and the results clearly indicated the remarkable performance of this controller and its learning algorithm. In addition, the result of comparing the performance of this controller with a genetically tuned classical PID controller has shown the superiority of the PID-like ANFIS controller.
\end{abstract}

Keyword: ANFIS; Classical PID controller; Genetic algorithms; Neuro-fuzzy systems. 